\title{
Corela
}

Cognition, représentation, langage

6-2 | 2008

Vol. $6, n^{\circ} 2$

\section{Effet de la compréhension de l'énoncé sur la résolution des problèmes arithmétiques}

\section{Sylvie Akiguet-Bakong}

\section{OpenEdition}

\section{Journals}

Édition électronique

URL : http://journals.openedition.org/corela/300

DOI : $10.4000 /$ corela.300

ISSN : 1638-573X

Éditeur

Cercle linguistique du Centre et de l'Ouest - CerLICO

Référence électronique

Sylvie Akiguet-Bakong, « Effet de la compréhension de l'énoncé sur la résolution des problèmes arithmétiques », Corela [En ligne], 6-2 | 2008, mis en ligne le 15 décembre 2008, consulté le 19 avril 2019. URL : http://journals.openedition.org/corela/300 ; DOI : 10.4000/corela.300

Ce document a été généré automatiquement le 19 avril 2019

\section{(c) (i) (3) (2)}

Corela - cognition, représentation, langage est mis à disposition selon les termes de la licence Creative Commons Attribution - Pas d'Utilisation Commerciale - Partage dans les Mêmes Conditions 4.0 International. 


\title{
Effet de la compréhension de l'énoncé sur la résolution des problèmes arithmétiques
}

\author{
Sylvie Akiguet-Bakong
}

\section{Introduction}

1 La compétence en arithmétique semble relever d'habiletés appartenant à un domaine plus vaste à savoir celui de la numération (cf. Fayol, Camos \& Roussel, 1997 ; Fayol, 2002). Il s'agit en fait de savoirs-faire ayant trait au comptage ainsi qu'à sa mise en oeuvre à travers des procédures d'addition, de multiplication, de soustraction et de division notamment dans le cadre de problèmes additifs. Ce type de problèmes a déjà fait l'objet d'une classification qui présente l'avantage d'en clarifier la nature. Riley, Greeno et Heller en sont les auteurs, ils ont en effet proposé en 1983 de regrouper les problèmes additifs en trois catégories à savoir :

- la catégorie «réunion" portant sur des situations statiques: «X a 3 bonbons. $Y$ a 4 bonbons. $\mathrm{X}$ et $\mathrm{Y}$ ont ensemble 7 bonbons ». Les problèmes consistent à trouver le total ou un état partiel ;

- la catégorie "changement " décrivant une transformation appliquée à un état initial et aboutissant à un état final « $\mathrm{X}$ avait 3 bonbons. Il en a gagné $4 . \mathrm{X}$ a maintenant 7 bonbons ». L'inconnue peut concerner l'état initial, la transformation (additive ou soustractive) ou l'état final ;

- la catégorie « comparaison » comparant des quantités statiques à l'aide de formules du type « plus que / moins que ». « X a 3 bonbons. Y a 7 bonbons. Y a 4 bonbons de plus que $\mathrm{X}$ ». Les problèmes consistent à trouver la grande partie, la petite partie, ou la différence.

Les connaissances actuelles relatives à l'instauration des habiletés numériques chez l'enfant font état d'une progression de la simple sensibilité à la différence numérique entre deux collections d'objets chez des enfants d'âge préscolaire dans des situations non 
verbales vers la mise en place d'une aptitude à la résolution de problèmes additifs chez des enfants d'âge scolaire.

Concernant les progrès liés à la sensibilité numérique chez l'enfant, des recherches empiriques (entre autres, Huttenlocher, Levine et Jordan, 1994 ; Lipton et Spelke, 2003 ; Mc Crink et Wynn, 2004) ont déjà permis de constater que dès la plus tendre enfance (6 mois, 9 mois, ou 2 à 4 ans) l'enfant est sensible à la différence numérique entre deux classes d'objets. L'attention des chercheurs est alors focalisée sur les réactions (durée de fixation du regard) de l'enfant face à des situations inattendues voire bizarres qui selon les auteurs témoignent de la capacité de discrimination de l'enfant et donc de sa sensibilité numérique.

4 C'est ainsi que Lipton et Spelke (2003) ont mené une étude visant à démontrer les capacités de discrimination de huit éléments sonores naturels (cloches, gazouillements, bourdonnement) chez 16 nourrissons (8 garçons et 8 filles ) âgés en moyenne de 6 mois. Avec un dispositif expérimental comportant deux haut-parleurs, l'un placé à la droite du sujet et l'autre à sa gauche, les auteurs ont débuté leur expérience par une phase de familiarisation du sujet à des séquences sonores identiques de six sons, fournit alternativement dans chaque haut-parleur. La phase expérimentale proprement dite a consisté à faire varier les séquences sonores produites dans l'un et l'autre haut-parleur (4 sons contre 8 sons ou bien 8 sons contre 16 sons). Les résultats obtenus ont indiqué qu'une majorité de nourrissons ( $75 \%$ a tourné plus longtemps la tête en direction des séquences sonores nouvelles fournissant ainsi une preuve de discrimination réussie.

Dans une autre expérience visant à mesurer la finesse de la discrimination chez les mêmes nourrissons que précédemment, les auteurs précités ont réduit la différence numérique entre deux séquences sonores de deux éléments soit 8 sons contre 12 sons. Les résultats n'ont indiqué aucune discrimination de la part des nourrissons. En revanche, la même expérience proposée à des nourrissons de 9 mois a fourni des résultats satisfaisants. Autrement dit les capacités de discrimination des nourrissons ont augmenté avec l'âge.

6 Pour leur part, Mc Crink et Wynn (2004), ont étudié la capacité à faire des calculs simples chez 26 nourrissons, des deux sexes, âgés en moyenne de 6 mois et répartis en deux groupes (un groupe addition et un groupe soustraction). Le dispositif expérimental a permis de placer les sujets devant un écran d'ordinateur et de les exposer, soit à la procédure d'addition, soit à celle de soustraction. Dans le cas de l'addition, les sujets ont assisté à la scène suivante : descente de cinq objets vers le bas de l'écran cachés peu après par un cache oculaire qui les recouvre entièrement. Puis, émergence et disparition de cinq objets additionnels derrière le cache oculaire. Dans le cas de la soustraction, le nombre d'objets dissimulés derrière le cache oculaire a été doublé, soit dix objets. La scène a été alors la suivante : déplacement de cinq objets en dehors du cache puis sortie de ces objets de l'écran. Dans les deux cas, le cache s'est laissé tomber pour laisser apparaitre soit cinq, soit dix objets, et ainsi le nourrisson a eu à découvrir, soit un événement impossible, soit un événement possible. Les auteurs ont constaté, d'une part, que les sujets exposé à la scène d'addition ont eu des durées de fixation plus longues lorsque cinq objets apparaissaient (10.28 s) que lorsqu'il y en avait dix (7.35 s). D'autre part, les enfants ayant assisté à la scène de soustraction ont plus longuement fixé les écrans finaux avec 10 objets (9.13s) que ceux avec 5 objets (8.00s). Mc Crink et Wynn (ibid.) en ont conclu que ces performances, relevant de la sensibilité numérique chez l'enfant, témoignaient ainsi de leur aptitude précoce à faire des calculs simples. 
7 Il faut savoir qu'au-delà de cette aptitude précoce de l'enfant à effectuer des activités numériques, notamment sur un petit nombre de collections d'objets n'excédant pas 10, il y a la difficulté introduite plus tard dans ce type d'activités par leur expression verbale dans les problèmes additifs rencontrés en milieu scolaire par exemple.

La recherche des causes à l'origine de cet état de fait a permis la mise en évidence de deux principales sources de difficultés pour les enfants : le codage verbal et la modélisation. A propos du codage verbal, Fayol, Camos et Roussel (2000) qui entendent par codage verbal le fait d'attribuer un nom à un nombre (lexicalisation), considèrent que celui-ci demande un effort d'apprentissage à l'enfant du fait du caractère arbitraire (l'arbitraire du signe linguistique de Saussure 1916) et conventionnel de l'association nom-nombre.

Duclosson (2004) évoque, pour sa part, une difficulté à modéliser les problèmes additifs chez l'enfant et à se représenter ainsi correctement la situation décrite verbalement par le problème (énoncé). L'auteur ajoute que la résolution d'un problème additif ou arithmétique implique au plan cognitif deux types de calculs : un calcul relationnel lié au traitement sémantique de l'énoncé (recherche de l'inconnue) et un calcul numérique relatif à l'identification de la procédure de résolution et à son application.

Il faut toutefois, relever que la procédure de résolution de tout problème additif exige une hiérarchisation de ces deux types de calculs, le relationnel précédent le numérique. De ce fait la difficulté éprouvée par les enfants dans la mise en place du calcul relationnel conduit à un calcul numérique erroné. C'est ce qu'a révélé Coquin-Viennot (2001) dans une étude visant à vérifier l'impact du traitement sémantique de l'énoncé sur la qualité de la résolution d'un problème arithmétique. L'auteur a présenté à 73 élèves de niveau Cours Moyen deuxième année ( $\mathrm{CM} 2$ ) un problème arithmétique dont l'énoncé avait subi quelques modifications comparativement aux énoncés de problèmes habituellement traités en milieu scolaire.

11 Ainsi, la question posée par cet énoncé était, soit attendue, soit inattendue, comparativement au récit de l'ensemble de l'énoncé. De plus cette question a eu deux positions dans l'énoncé : soit au début de l'énoncé, soit à la fin de l'énoncé. L'analyse des performances des sujets de cette recherche a indiqué que la bonne performance était liée à la position de la question du problème en début d'énoncé plutôt qu'en fin d'énoncé notamment dans le cas des questions attendues. Ce résultat suggère l'existence d'un impact majeur de la qualité du traitement sémantique (linguistique) de l'énoncé sur la résolution des problèmes arithmétiques.

En somme les recherches précitées suggèrent l'idée selon laquelle la résolution des problèmes arithmétiques reposerait sur un aspect cognitif majeur à savoir la capacité du sujet à se représenter les données en présence, qu'elles soient numériques ou sémantiques (linguistiques).

\section{Objectifs et Hypothèses}

13 La présente recherche a pour objectif de vérifier l'impact du niveau de compréhension de l'énoncé sur la résolution des problèmes arithmétiques chez de jeunes enfants scolarisés. La perspective choisie s'inspire des travaux antérieurs relatifs au traitement sémantique de l'énoncé mais ne se limite pas à la position (avant ou après l'énoncé) de la question finale d'un problème, très largement traitée dans la littérature (cf. Fayol, Camos et Roussel; 2000 pour une revue de question). Au contraire, dans la présente étude il est 
admis que la recherche de la compréhension des difficultés rencontrées par les élèves du primaire notamment lors de la résolution des problèmes arithmétiques gagnerait à être complétée par une analyse de la séquence d'actions de cette résolution. Il s'agit de pister, au plan cognitif, la manière dont le sujet parvient à détecter le raisonnement sous-tendu par le lien établi entre l'énoncé et sa question.

Concrètement, il est question de démontrer que l'énoncé en tant que récit (Adam, 1992; Fayol, 1986) comporte un certain nombre d'événements orientés vers un même but. Le traitement de cet énoncé par le sujet exige de sa part une aptitude à pouvoir se construire une représentation (Kintsch, 1976) ou un modèle mental (Johnson-Laird, 1983) de chacun des événements décrits par le récit ainsi que leur cohérence interne par rapport au problème posé. Le sujet face à un problème arithmétique a donc à faire un premier effort mental de représentation de la cohérence interne du problème qui dépend de la représentation détaillée de chacun des événements présentés dans l'énoncé. La qualité de cette représentation relative à la cohérence du récit conditionne une deuxième représentation relative à la procédure de calcul à mettre en œuvre pour résoudre le problème posé. Compte tenu de cette analyse, l'hypothèse de travail de la présente recherche est formulée ainsi qu'il suit : La résolution d'un problème arithmétique serait limitée par la difficulté à pouvoir repérer la cohérence interne de l'énoncé à travers la prise en compte des données (événements) qui y sont indiquées.

\section{Méthode}

\section{Population}

1540 sujets ont pris part à cette expérience : 20 garçons et 20 filles dont l'âge, compris entre 8 et 12 ans, correspond à un âge moyen de 9 ans 4 mois. Il s'agit d'enfants régulièrement scolarisés dans une même école de la banlieue de Libreville en classe de troisième année du cycle primaire (C.E.1). Ces quarante sujets ont été obtenus en sélectionnant, dans les quatre $\mathrm{CE} 1$ de l'école retenue, dix élèves (les 5 meilleurs élèves et les 5 moins bons élèves en arithmétique) des deux sexes. Ces élèves ont ensuite été répartis en deux groupes expérimentaux, puis parfaitement équilibrés sur le plan du sexe (autant de filles que de garçons) et sur le plan du niveau en arithmétique (autant de très bons élèves que de moins bons en arithmétique).

\section{Matériel}

Quatre problèmes arithmétiques tirés d'un ouvrage au programme dans le système scolaire des élèves de cette expérience ont été retenus (cf. petit encadré ci-après). Selon la catégorisation proposée par Riley \& coll. (1983), au plan de la résolution, deux de ces problèmes appartiennent à la catégorie « changement » (problèmes 1 et 4) tandis que les deux autres problèmes appartiennent, l'un à la catégorie « comparaison » (problème 2) et l'autre à la catégorie " réunion » (problème 3 )

Problème 1: Allang et son père vendent des œufs. Ils en ont 360. Avec Allang, tu ranges les œufs dans 10 paquets. Calcule la quantité d'œufs que doit contenir chaque paquet.

Problème 2: A Mouila il y a 8345 enfants. Seuls 4286 sont vaccinés contre la rougeole. Le nombre d'enfants qui ne sont pas vaccinés contre la rougeole à Mouila est de : 
Problème 3 : Au marché, Obone a vendu 28 beignets le matin et 34 l'après-midi.

Combien de beignets Obone a t-elle vendu dans la journée? posé par chaque énoncé, un petit questionnaire leur a été proposé, après la lecture de l'énoncé et avant le traitement de chaque problème. Ce petit questionnaire a été élaboré en se focalisant sur le contenu sémantique de l'énoncé. Il s'est agi plus précisément, d'interroger les sujets sur chacune des informations données dans l'énoncé en suivant l'ordre de leur apparition dans la progression de cet énoncé. Par exemple : le lieu du récit de l'énoncé, le nom du protagoniste, le nom de l'objet à vendre, ce que la question finale demande de trouver. Dans la dernière question de ce questionnaire, il a été demandé au sujet d'indiquer l'opération appropriée à la résolution du problème posé par l'énoncé parmi les quatre opérations à sa connaissance, à savoir : l'addition (+), la soustraction(-), la multiplication(x) et la division( :).

Deux conditions expérimentales ont été obtenues en faisant varier la présentation de l'épreuve. En effet, dans une condition, l'énoncé du problème suivi du questionnaire a été présenté sur un seul et même support-papier et dans l'autre condition la présentation de ces deux éléments (énoncé et questionnaire) a été faite sur deux supports-papiers distincts mais solidement agrafés afin de conserver l'ordre précité (énoncé suivi du questionnaire).

En somme, comme matériel cette expérience a nécessité :

- 4 problèmes

- 4 questionnaires

Tous ont fait l'objet d'une saisie sur ordinateur à l'aide d'un traitement de texte(Microsoft Word) et d'une impression sur papier de format A4.

- 1 chronomètre.

\section{Procédure}

Pour permettre aux sujets d'effectuer les épreuves de cette expérience un mode de passation de type collectif a été retenu. Ainsi, chaque groupe expérimental de 20 sujets une fois installé dans une salle de classe de leur école, à raison d'un sujet par table-banc, a reçu des mains de l'expérimentateur l'épreuve à traiter à savoir une série de quatre problèmes sur quatre feuilles empilées les unes sur les autres. Puis, la consigne a été donnée oralement. Dans une des conditions expérimentales (avec aide mémoire), cette consigne était la suivante : "Vous avez chacun quatre petits problèmes. Chaque problème est suivi par quelques questions. A mon signal (battement des mains) lisez bien le problème posé sous vos yeux, répondez aux questions posées à la suite, puis posez et effectuez l'opération juste pour bien résoudre ce problème dans l'espace vide laissé pour cela.. Attention! Vous pouvez relire l'énoncé du problème, de temps en temps, si vous l'avez oublié! A mon deuxième signal vous passez au problème suivant et ainsi de suite jusqu'à ce que je vous fasse signe d'arrêter d'écrire » Dans l'autre condition expérimentale (sans aide mémoire), cette consigne a été légèrement modifiée comme suit: "Vous avez chacun quatre petits problèmes. La feuille de chaque problème est collée à une autre feuille où se trouvent quelques questions. A mon signal lisez l'énoncé d'un problème pendant quelques minutes(3minutes), ensuite tournez la page en cachant l'énoncé sous la feuille des questions (Attention il ne faudra plus retourner cette feuille pour lire l'énoncé) et répondez aux questions posées, puis posez et effectuez l'opération juste pour bien résoudre ce problème dans l'espace vide laissé pour cela. Enfin à mon signal arrêtez d'écrire ».

Corela, 6-2 | 2008 
Il faut savoir que dans chacune des deux conditions précitées, la durée de passation imposée a été de 10 minutes.

\section{Variables dépendantes et analyse de données} $\mathrm{S}_{40}<\mathrm{C}_{2}>{ }^{*} \mathrm{~T}_{2} * \mathrm{P}_{4}$ la lettre (S) est mise pour le facteur sujet, elle est affectée de l'indice 40 correspondant à la taille de l'échantillon de cette expérience. L'ensemble de cet échantillon est soumis à l'effet d'un facteur expérimental nommé «condition » dont la variabilité se traduit par la présence ou l'absence d'un aide mémoire pendant la résolution de la tâche, d'où la lettre (C) affectée de l'indice 2. Ce facteur "condition " emboîte deux autres facteurs, à savoir la tâche $(\mathrm{T})$ et le problème $(\mathrm{P})$. Le facteur tâche a pour modalités la compréhension (tâche de traitement des questions se rapportant à un problème donné) et la résolution (tâche de résolution d'un problème arithmétique donné). Quant au facteur problème, il compte quatre modalités relatives au nombre de problèmes arithmétiques traités par tous les sujets de cette recherche.

L'analyse de l'effet des facteurs expérimentaux précités sur la performance des sujets a donné lieu à l'identification de deux variables dépendantes à savoir: le score de compréhension du questionnaire et le score de résolution de problème.

Pour ce qui concerne le score de compréhension, il a été obtenu, pour chaque sujet, en divisant le nombre de ses bonnes réponses par le nombre total de questions à traiter. Il faut savoir que chaque problème à résoudre compte en moyenne 7 questions. Le fait de répondre correctement à l'une d'elle correspond à une (1) bonne réponse dans le cas contraire c'est une mauvaise réponse.

23 Le score relatif à la qualité de la résolution d'un problème, a quant à lui été obtenu en attribuant à chaque sujet un score soit égal à 1 lorsque la solution adéquate au problème a été trouvée, soit égal à 0 lorsque la solution proposée était erronée.

Afin de vérifier l'effet des trois facteurs expérimentaux de cette recherche sur les variables dépendantes retenues, une analyse de variance a été effectuée à l'aide du logiciel « Statistical Package for the Social Sciences » (S.P.S.S.)

\section{Résultats}

\section{Effet de la condition expérimentale}

Le plan d'analyse de variance retenu pour l'étude du facteur « condition » est le suivant :

$\mathrm{S}_{40}<\mathrm{C}_{2}>\mathrm{xP}_{4}$. Les résultats concernant ce facteur sont présentés suivant les deux variables dépendantes de cette recherche.

L'analyse des données obtenues indique que le facteur " condition expérimentale » a eu un impact sur la performance des sujets (cf. figure $n^{\circ} 1$ ), qu'il s'agisse de taux de compréhension $F(1,158)=4,91 ; p<.05$ ou de taux de résolution $(1,158)=4,62 ; p>05)$. Les sujets ayant eu à effectuer leur tâche en présence du texte de l'énoncé du problème à résoudre (condition "aide mémoire») obtiennent des scores moyens $(0,54$ en compréhension et 0,48 en résolution) supérieurs à ceux ( 0,44 en compréhension et 0,32 en résolution) de leurs camarades privés d'énoncé (condition "sans aide mémoire »). Autrement dit, le fait de maintenir la présence de l'énoncé à contribué doublement à la 
bonne performance des sujets : dans la compréhension du problème posé comme dans sa résolution.

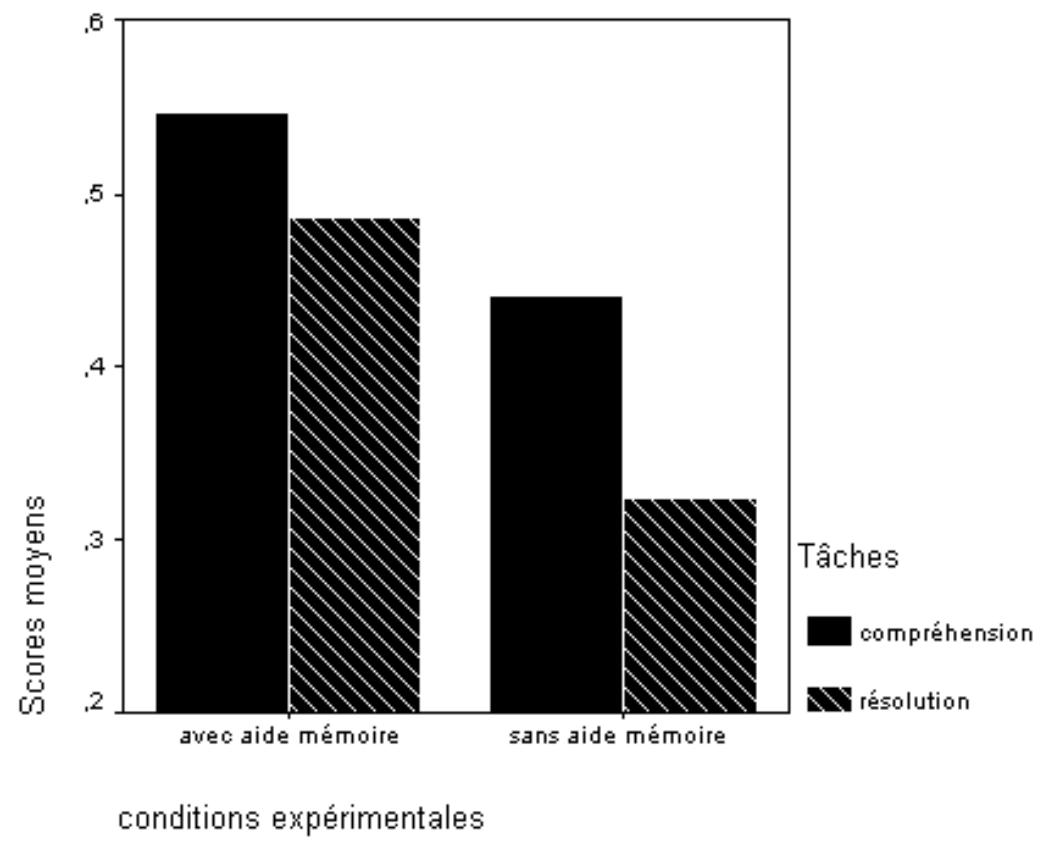

\section{Figure 1: Scores moyens en compréhension et en résolution selon la condition expérimentale}

\section{Effet de la tâche}

Concernant l'étude de l'effet de la tâche, le plan d'analyse de variance adopté a été le suivant: $\mathrm{S}_{40}<\mathrm{C}_{2}>* \mathrm{~T}_{2} * \mathrm{P}_{4}$. Un effet significatif du facteur « tâche » sur la performance des sujets est également à noter: $F(1,318)=3,906 ; \mathrm{p}<.05$, le score moyen obtenu en compréhension $(0,49)$ des questions relatives à l'énoncé étant supérieur à celui obtenu pour la résolution $(0,40)$ du problème. En d'autres termes, compréhension et résolution sont ainsi apparues comme deux tâches ne sollicitant pas les mêmes compétences chez les enfants. Ce qui a pour conséquence de révéler que la tâche de compréhension (traitement sémantique du problème) est plus facile que celle de résolution (traitement numérique du problème). 


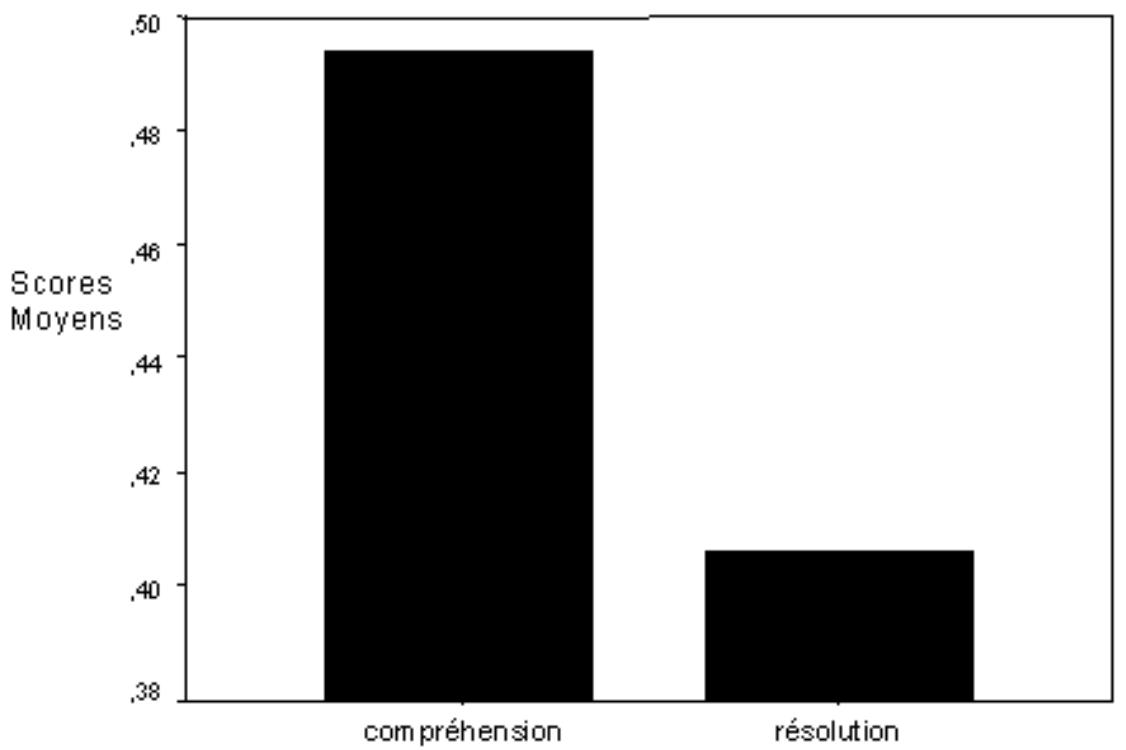

Tâches

\section{Figure 2: Scores moyens en fonction de la tâche}

\section{Effet du problème}

Suivant le même plan d'analyse de variance adopté : $\mathrm{S}_{40}<\mathrm{C}_{2}>\mathrm{x} \mathrm{P}_{4}$, lorsqu'il s'agit de compréhension aucun effet significatif du problème sur la performance des sujets (scores moyens du problème 1 au problème $4: 0,42 ; 0,45 ; 0,55 ; 0,54)$ n'est à signaler $(F(3,156)=$ 1,$89 ; \mathrm{p}=.13$, n.s.). En revanche, l'analyse des productions des sujets relatives à la résolution des problèmes indique un effet significatif de la variation de ces problèmes sur la performance des sujets : $F(3,156)=3,45 ; p<.05$. En fait, les quatre problèmes donnés successivement (cf. les chiffres 1,2,3,4 affectés à ces problèmes dans la figure $n^{\circ} 3$ ) à traiter aux sujets n'ont pas présenté, pour eux, le même niveau de difficulté. Suivant les scores moyens obtenus par ces sujets lors de leur résolution, l'ordre de difficulté de ces problèmes (cf. figure $\left.\mathrm{n}^{\circ} 3\right)$, du plus difficile au plus facile, est le suivant : problème $2(\mathrm{~m}=$ $0,22)$, problème $1(m=0,37)$, problème $4(m=0,47)$, problème $3(m=0,55)$. En fait le problème traité en deuxième position par les sujets (problème 2 de la catégorie « comparaison ») semble avoir été le plus difficile, tandis que celui traité en troisième position (problème 3, catégorie « réunion ») a été le plus facile. 


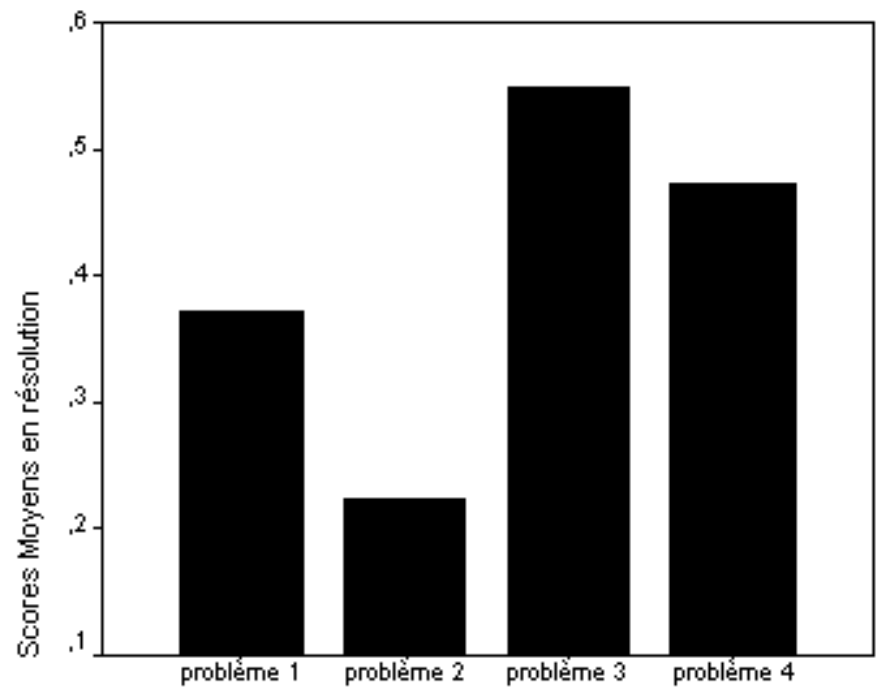

Problèmes

\section{Figure 3 : Scores moyens de résolution en fonction des problèmes}

De plus, un seul des contrastes calculés est significatif . Il s'agit de celui opposant, en résolution, le problème 3 au problème $2(\mathrm{~F}(3,152)=3,45 ; \mathrm{p}<.05)$.

\section{Discussion}

L'hypothèse de travail «La résolution d'un problème arithmétique serait limitée par la difficulté à pouvoir repérer la cohérence interne de l'énoncé à travers la prise en compte des données (événements) qui y sont indiquées » ayant guidé la présente recherche a été vérifiée. Puisqu'il s'est avéré que la qualité du traitement de l'énoncé est un facteur déterminant dans la résolution d'un problème arithmétique.

De plus, au terme de cette étude, quelques constats peuvent être faits. D'abord en ce qui concerne l'effet du facteur « condition ». Son analyse révèle un fait important relatif aux capacités de la mémoire de travail chez les élèves examinés. En effet, il a été constaté que lorsque les capacités de la mémoire de travail sont soutenues voir amplifiées (condition " avec aide mémoire ») par la présence prolongée du matériau (énoncé du problème) nécessaire à la résolution de la tâche, les sujets aboutissent à une performance de meilleure qualité, en compréhension comme en résolution, que lorsque ces capacités sont limitées (condition "sans aide mémoire »). La disponibilité du matériau agit donc, dans le cas présent de résolution de problèmes arithmétiques comme un facteur renforçateur des capacités de traitement de la mémoire de travail chez les élèves qui améliore leur niveau de compréhension de l'énoncé et par conséquent leur niveau de résolution du problème posé par cet énoncé.

Ensuite, la différence de performance constatée entre la tâche de compréhension et la tâche de résolution, la première ayant été plus facile à traiter que la deuxième, indique que des connaissances de natures différentes ont été sollicitées chez les sujets. Il s'agit pour la tâche de compréhension de connaissances dites déclaratives (faits ou 
informations descriptibles verbalement et dont l'organisation est contrôlable par le sujet selon Lemaire, 1999). Et, pour la tâche de résolution, il est question de connaissances dites procédurales, autrement dit de celles qui, selon Reed (1999) lient les actions aux buts. De plus, en ce qui concerne ces deux types de connaissances, les sujets de cette expérience n'ont pas le même niveau de compétence. En d'autres termes, les connaissances déclaratives et celles procédurales n'auraient pas le même rythme d'acquisition chez ces sujets. Ces sujets auraient moins de difficultés à faire le traitement sémantique d'un énoncé que le traitement numérique qu'implique cet énoncé à travers la mise en œuvre d'une procédure de résolution arithmétique. Ce résultat relatif à la différence de performance constatée en fonction des tâches (compréhension versus résolution) est en conformité avec les idées émises par Duclosson (2004) faisant état de l'existence d'un double calcul (relationnel et numérique) dans tout problème arithmétique.

Enfin, les données relatives à l'effet du "problème » sur la performance des sujets de cette expérience corroborent l'idée d'une catégorisation des problèmes additifs émise par Riley, Greeno et Heller (1983). En fait s'il est possible de créer des catégories parmi les problèmes arithmétiques (réunion, changement, comparaison) cela suppose au plan cognitif des contraintes différentes pour le sujet et partant des niveaux de performance différents. C'est ce que révèlent les résultats de la présente recherche, notamment en ce qui concerne l'analyse de l'effet du problème sur la performance des sujets. Il s'est avéré que les quatre problèmes donnés à traiter à chaque sujet n'ont pas recueilli des niveaux de performance identiques. Au contraire, certains problèmes sont apparus plus faciles (problèmes 3 de la catégorie "réunion») à traiter que d'autres ( problèmes 2 de la catégorie « comparaison »).

$\mathrm{Au}$ vu de l'ensemble des résultats obtenus, la compréhension de l'énoncé est un facteur important pour la résolution d'un problème arithmétique. La question de l'identification par les sujets du lien (cohérence) existant entre les différentes informations que comporte un énoncé et le problème que pose cet énoncé s'est avérée pertinente. En fait les difficultés rencontrées par les écoliers pour traiter les problèmes arithmétiques sont certainement de plusieurs ordres, mais en ce qui concerne l'analyse de l'énoncé, la présente recherche a montré que le manque de disponibilité physique et partant mentale des données de l'énoncé, par exemple dans le cas d'une lecture hâtive de l'énoncé, peut être un handicap à la résolution d'un problème. De même, la différence de nature entre des problèmes distincts ainsi que la différence des compétences (langagière et numérique) exigées par un problème donné peuvent constituer des limites à la résolution des problèmes arithmétiques. Il faut d'ailleurs ajouter à ces limites, un fait non pris en compte par la présente étude mais non moins important, à savoir l'émotion ou l'anxiété occasionnée par la difficulté à se représenter le problème à résoudre.

En conséquence, cette étude qui n'a pas eu pour objet de donner des leçons aux pédagogues de l'arithmétique, peut toutefois suggérer à ces derniers de se focaliser sur l'ensemble des compétences de diverses natures impliquées dans l'apprentissage de l'arithmétique, notamment dans la conception des manuels, afin d'offrir un cadre approprié, sorte d'ergonomie cognitive à cet apprentissage. 


\section{BIBLIOGRAPHIE}

Adam, J.M. (1992). Les textes : types et prototypes. Récit, description, argumentation, explication et dialogue. Paris : Nathan.

Aye, F. et Lehalle, H. (2006). Contexte culturel et acquisitions numériques. L'exemple des nombres négatifs en France et à la Réunion. Enfance, vol. 58, n² 2, pp. 159-168.

Coquin-Viennot, D. (2001). Problèmes arithmétiques verbaux à l'école : pourquoi les élèves ne répondent-ils pas à la question posée. Enfance, vol. 53, n² 2, pp. 181-196.

De Saussure, F (1961). Cours de linguistique générale(5 $5^{\text {ème }}$ édition). Paris, Payot.

Duclosson, N. (2004). Représentation des connaissances dans l'EIAH AMBRE-add. Technologies de l'Information et de la Connaissance dans l'Enseignement Supérieur et l'Industrie. Compiègne : Université de Technologie de Compiègne. pp. 164-171.

Fayol, M. (2002). Le facteur verbal dans les traitements numérique : perspective développementale, in J. Bideau \& H Lehalle (éd.). Traité des sciences cognitives : le développement des activités numériques chez l'enfant (pp. 151-173). Paris : Hermes.

Fayol, M. ; Camos, V. ; Roussel, J-L. (2000). Acquisition et mise en œuvre de la numération par les enfants de 2 à 9 ans. In M. Pesenti et X. Seron (Eds.). Neuropsychologie du calcul et du traitement des nombres (pp. 33-58). Marseille : Solal.

Huttenlocher, J., Jordan, N.C., \& Levine, S. C. (1994). A mental model of early arithmetic. Journal of Experimental Psychology: General, 123, pp. 284-296.

Johnson-Laird, P. (1983). Mental Models. Cambridge, MA: Harvard University Press.

Kintsch, W. (1976). Bases conceptuelles et mémoire de texte. Bulletin de Psychologie, numéro spécial : la mémoire sémantique, pp. 327-334.

Lipton, I. S. et Spelke, E. S. (2003). Origins of number sense: large-number discrimination in human infants. Psychological Science, vol 14, n 5. pp. 396-401.

Lemaire, P. (1999). Psychologie Cognitive. Paris: Deboeck Université.

Mc Crink , K. et Wynn, K. (2004). Large-number addition and subtraction by 9-month-old infants. Psychological Science, vol 15, $\mathrm{n}^{\circ} 11$, pp. 776-781.

Reed, S. K. (1999). Cognition. Paris: De Boeck Université.

Riley, M.S., Greeno, J. G., \& Heller, J.I. (1983). Development of children's problem-solving ability in arithmetic. In H.P. Ginsburg (Ed.), The development of mathematical thinking. New-York: Academic Press.

Van Dijk, T. A. (1982) Attitude et compréhension de textes. Bulletin de Psychologie, vol. 35, 356, pp. 557-560.

Van Dijk, T.A. \& Kintsch, W. (1983). Strategies of discourse comprehension. New-York: Academic Press.

Van Nieuwenhoven, C. et Collet, M. (2000). Le comptage et les premiers apprentissages numériques. Actes du $1^{\mathrm{er}}$ Congrès des chercheurs en éducation. 24-25 mai,Bruxelles : Ministère de la communauté française. 
Vincent, Suzanne (1997). Des conduites d'élèves en construction - le cas de figure des relations multiplicatives. Education et francophonie. (revue scientifique virtuelle http://www.acelf.ca/c/ revue/), $\mathrm{n}^{\circ} 1$, vol. 25 .

\section{RÉSUMÉS}

La présente recherche a eu pour objectif de vérifier l'impact du niveau de compréhension de l'énoncé sur la résolution des problèmes arithmétiques chez de jeunes enfants scolarisés (niveau CE1 : $3^{\text {ème }}$ année primaire). A l'aide d'un paradigme expérimental permettant, tout à la fois, de raccourcir ou de prolonger la durée de la présence de l'énoncé en mémoire de travail, de confronter le niveau de compréhension de l'énoncé à la qualité de la résolution du problème posé par cet énoncé, et d'éprouver la performance en arithmétique des sujets sur quatre problèmes différents, cette recherche a permis l'obtention d'un résultat particulièrement intéressant au regard de l'objectif visé. En effet cette recherche a permis de constater que lorsque les conditions de traitement de l'énoncé sont favorables à une meilleure compréhension de ce dernier la performance en arithmétique des sujets s'améliore.

Impact of the arithmetic text problem comprehension on its resolution. This study focused on the impact of the arithmetic text problem comprehension level on its resolution by young children from the elementary school (level 3 of the primary school). With an experimental paradigm that combines the capacity to shorten or extend the duration of the text problem presence in the working memory, the confrontation of the text comprehension level to its resolution quality and that tests the children arithmetic ability on four problems, this research led to a particularly interesting result according to the objective focused on. In fact, it shown that when the treatment conditions of an arithmetic text problem allow a better comprehension, the children resolution performance improves.

\section{INDEX}

Mots-clés : cognition, arithmétique, problèmes additifs, compréhension de texte, connaissances procédurales, connaissances déclaratives, numération

Keywords : cognition, arithmetic problems, text comprehension, procedural knowledge, declarative, knowledge, numerical computational ability

\section{AUTEUR}

\section{SYLVIE AKIGUET-BAKONG}

Université Omar Bongo, Liberville (Gabon), Département de Psychologie, Centre de Recherches et d'études en Psychologie (C.R.E.P.) 